\title{
A Platform for 3G and Internet-Based Healthcare System
}

\author{
Xingzu Guo ${ }^{1,2, \text { a }}$, Xianxiang Chen ${ }^{2, b}$, Zhen Fang ${ }^{2, \text { c }}$, Shan hong, Xia, d \\ ${ }^{1}$ Graduate University, Chinese Academy of Sciences, Beijing 100049, China \\ ${ }^{2}$ State Key Laboratory of Transducer Technology, Institute of Electronics, Chinese Academy of Sciences \\ Beijing, China \\ \{ahdwgxz, $\left.{ }^{\mathrm{b}} \mathrm{cxxvac}\right\} @ 163 . c 0 m,\left\{{ }^{\mathrm{c}} \mathrm{zfang},{ }^{\mathrm{d}}\right.$ shxia\}@mail.ie.ac.cn,
}

\begin{abstract}
A java web-based platform for $3 G$ and internet-Based Healthcare System was designed to enable the guardian with chronic disease to get help. Apache Tomcat is used as web server and MySQL is used as database server. The healthcare system platform provides some functions such as querying physiologic data information, generating PDF format health information statistics report, consulting between experts. The physiologic data such as ECG and blood oxygen saturation (SPO2) obtained by using wearable physiologic monitors was uploaded to server database. The platform also has data analysis capabilities and makes preliminary assessment of some diseases.
\end{abstract}

Keywords-3G and internet technology; platform; Java Web; physiologic data;

\section{INTRODUCTION}

Aging of the world's population is becoming increasingly serious. The Global senior-aged people (individuals aged 65 years and older) accounted for 10 percent in 1998 and are projected 15 percent 2025. The increase will be most rapid for developing countries, which will have four times as many aged people in 50 years from now. According to data released by the National Bureau of Statistics of China, Chinese people who is over 60 years old in the total population of china exceeded 10 percent in 2000 and had reached 12.5 percent by $2009^{[1][2]}$. Population aging brings pressure to medical care.

In recent years, computer technology, electronic technology, communication technology are developing fast. This allows the device to have smaller volume, faster processing speed and more processing power. People's livings standards are improving .They are more concerned about their health and their requirements are also increasing.

The problem mentioned above can be partly solved by 3G and Internet-Based Healthcare System in which Internet-based Healthcare System platform is an important part. It can give the elderly a convenient query and consulting services as long as the Internet can be used .It also provide service for people who willing to use the Healthcare System platform such as, athlete, patients with heart disease and children et al.

In this paper we report an Internet-based Healthcare System platform. Section II gives concrete design process and realization method. Section III shows the work we have completed and how to use this platform. Future for more in-depth work will be described in the section IV.

\section{DESIGN AND IMPLEMENTATION}

A. Overview of $3 G$ and Internet-Based Healthcare System The architecture of $3 \mathrm{G}$ and Internet-Based Healthcare System is shown in Figure 1. People who wear wearable physiologic monitor ${ }^{[3]}$ get physiologic data and then transmitted the data to the android smart phone with Bluetooth module. These physiologic data can not only be displayed on the smart phone, but also be transmitted to the web server through $3 \mathrm{G}$ or WiFi. The collected physiological data can also be transmitted to a computer which has a USB Bluetooth dongle.

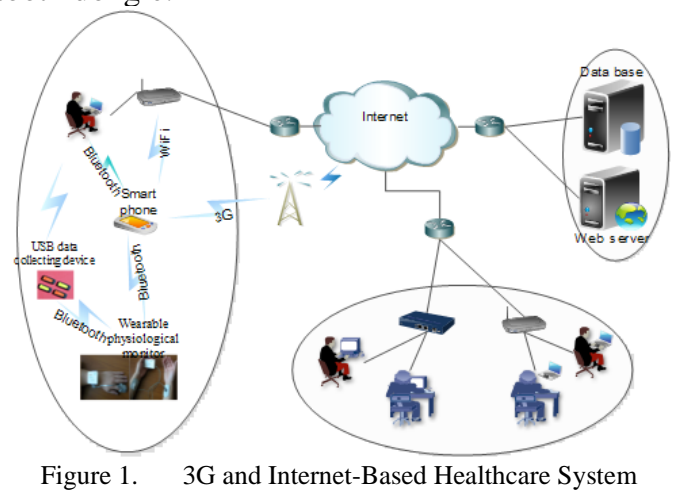

B. Platform design pattern and Functional Architecture

The Apache Tomcat ${ }^{[4]}$ was used as our web server that is an open source software implementation of the Java Servlet and JavaServer Pages (JSP). We adapted Model 2 design pattern which is shown in Figure 2 to design this platform [5]

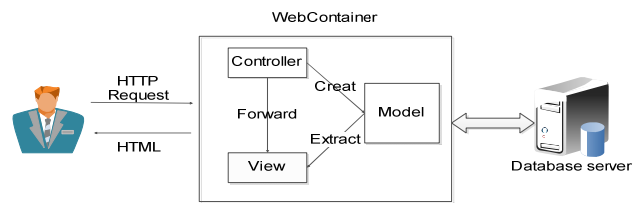

Figure 2. Platform design pattern

In Model 2, JavaBeans represents the model, JSP represents the view and Java Servlet represents the controller. Before functional architecture of the platform is defined, we analysis characteristics that the platform involves. People use wearable physiologic monitor to get physiologic data and then upload the data to the database server. When he wants to view the data, like ECG, he can log into the platform. Of course registering the information should be done first. The other operations are as follows: modifying the information, uploading file, consulting a 
doctor. Doctor would like to use the platform to answer some question that his patient raised and he also must register as a member. Doctor's operation is similar to the operation of the guardian (patient or consumer). Furthermore, we need to set a manager role. Through the above analysis we get Use-Cases for the platform, which was shown in Figure 3. And then it is easy to obtain a functional Architecture, shown in Figure 4, which is composed by five functional modules.
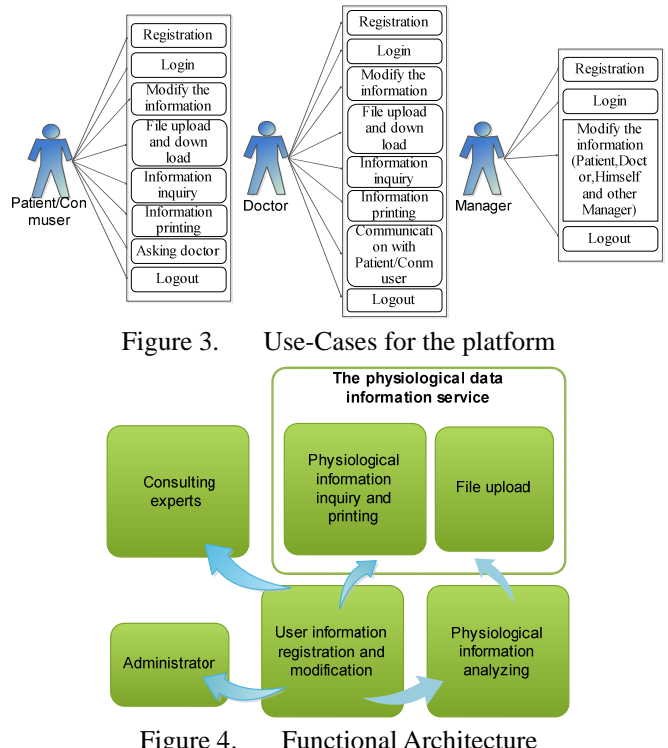

C. Modules Design and Implementation

The interaction between modules that are relatively independent refers to calling relationships between classes (as shown in Figure 4). Each module is in line with the model 2 design pattern. The benefit of this design is that the platform has very good extensibility in the future.

1) User information registration and modification module

Before completing the other modules, this module was designed at first. The guardian (patient or consumer) and expert (doctor) are allowed to register information. The information of the manager is stored in the database server. It is impossible to become a manager through registration unless setting up the information on the server side. Although one can't register to become a manager, he could modify his information as long as he already has been a manager. The function of the logging in and exiting has been realized on this module.

2) The physiologic data information service module

This module which is the core of the platform includes two sub-modules.

a) Physiologic information inquiry and printing

This sub-module's function is to provide users with physiologic data query and printing physiologic monitoring report. Here, we use two open source library that are JfreeChart and iText. JFreeChart is a Java chart library ${ }^{[6]}$. We use it to demonstrate physiologic parameters data that makes it easy for patient or doctor to identify difference with normal. ECG interface not only display ECG but also includes RR interval time series, QRS width, PR interval, QT interval and ST interval. The other interfaces, like SPO2 interface, display the characteristics of the data and include early warning function to determine whether it is normal. iText is the world's leading F/OSS PDF library and we use Java version to generate PDF documents for the printing interface $^{[7]}$. The relationship of the classes was shown in Figure 5: To the line a, ConnDB.java is responsible for connecting to the database and arrow represents some classes that are HeartBeancl.java, OxyBeancl.java, TemBeancl.java, PulseBeancl.java call ConnDB.java. To the line $b$, arrow pointing indicates that HeartBean.java is called by HeartBeancl.java and PrintheartServlet.java. To the line $\mathrm{f}$, HeartBeanclServlet.java, HeartBeanclRServ.java, PrintheartServlet.java, Pheanyser.java, Dheanyser.java, they all call HeartBeancl.java.

b) File upload

The purpose of this module is designed to facilitate the users to upload physiologic data files and download the resources they need. HttpServletRequest calls getInputStream() to get upload the data . Reading input data stream line by line, it is not difficult to find out not only the physiologic data but also some additional information such as the user name, the type of the physiologic parameters data and other useful information.

3) Physiologic signals analyzing module

It is important for the platform to have analysis functions. The analyzed data displayed in the query interfaces is generated by this module.

ECG analysis is more complicated than the other physiologic data analysis. Wavelet transformation algorithm was used to process ECG data. In order to be called conveniently in other classes, the function interface with the ECG characteristic analysis has been made. The function interfaces are as follows: 1) cbsw4 (int Id, int begin), obtain 4th decomposition level using cubic b-spline wavelets. 2) getR(int Id, int begin), obtain the peak of the R-waves. 3) getRR(int Id, int begin), obtain RR interval time series. 4) geteverchar(int Id,int begin), obtain P width, T width, QRS width, PR interval, QT interval, ST interval.

It is relatively simple for the other physiologic data analysis that the change is not intense to the short period data, generally forming a linear pattern.

4) Consulting experts module

This functional module has been designed to let the guardian communicate with doctor (expert) very conveniently. The doctor-patient dialogue content saved to a XML file is read utilizing JDOM which is a XML Java toolkit for rapid development of XML application ${ }^{[8]}$. Besides doctor-patient dialogue included in the XML file, there is some other information: file creation date, physiologic data category and system announcement. The format of the file creation date is "yyyyMMdd" but not "yyyyMMddhhmmss" because we save the data generated in a day to one XML document. Physiologic data category includes "IsPrivate" that represents one-to-one dialogue between the guardian and doctor, avoiding being seen by other guardians. 


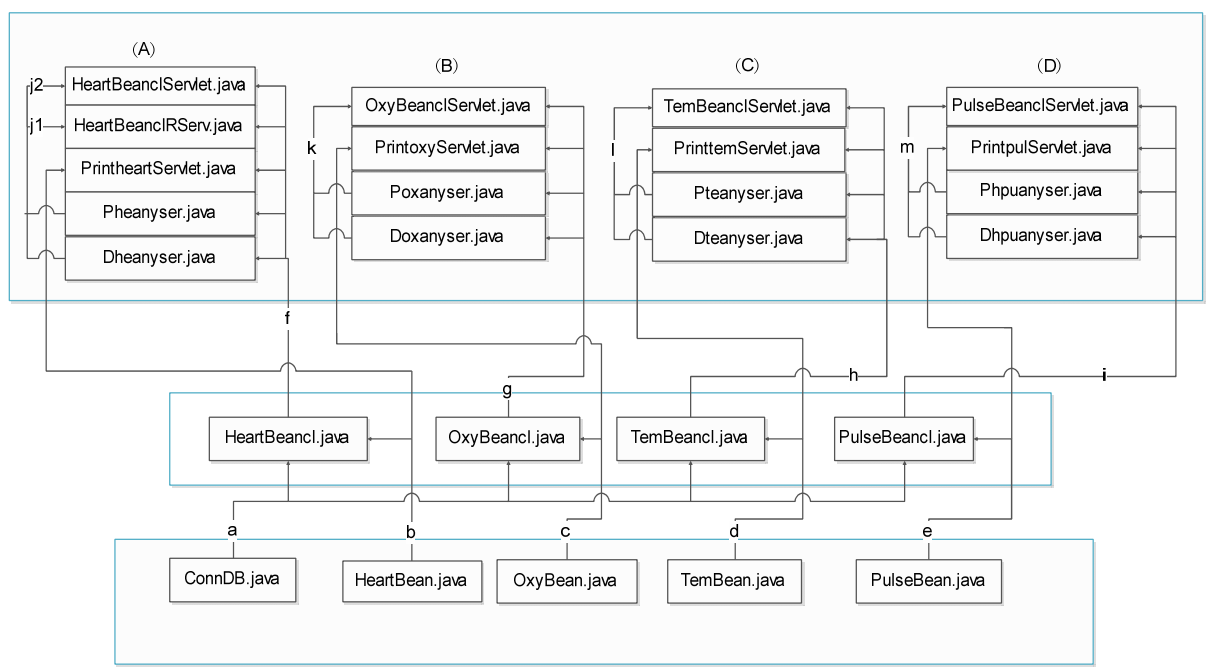

Figure 5. Classes’ relationship. (1) HeartBean.java, OxyBeancl.java, TemBeancl.java and PulseBeancl.java constitute model of Model 2 design pattern. (2) HeartBeancl.java, OxyBeancl.java, TemBeancl.java and PulseBeancl.java are responsible for processing registration information. (3) OxyBeanclServlet.java, PrintoxyServlet.java, Poxanyser.java and Doxanyser.java realize blood oxygen saturation graph and PDF document, the other part (C) and (D) are respectively responsible for realizing body temperature graph inquiry, printing body temperature report and pulse rate graph, printing pulse rate report.

5) Manager module

The function of this module is to view the registration information of all users, including the guardian (patient or customer), doctor (expert) and modify the information of manager itself. If you entered a person's Username (login name) in the search box, you can get his information and delete his information. This function is used only when the user forget to cancel his registration account that has been not used.

\section{Database Design and Implementation}

We designed and developed a relational database and implemented it through MySQL database management system (DBMS). Seven tables were designed such as the guardian registration information table, doctor (expert) registration information table, ECG data information table and blood oxygen data information table. There is a one-to-many binary relationship between the guardian and the physiologic data (include ECG, Blood oxygen saturation, Body temperature, Pulse rate) (as shown in Figure 6. a): The guardian may measure the same kind of physiologic data that may have different length at different time. There is a one-to-many binary relationship between manager and doctor (as shown in Figure 6.b). There is a one-to-many binary relationship between manager and the guardian (as shown in Figure 6.b). One manager can modify or delete many doctors or the guardian registration information.

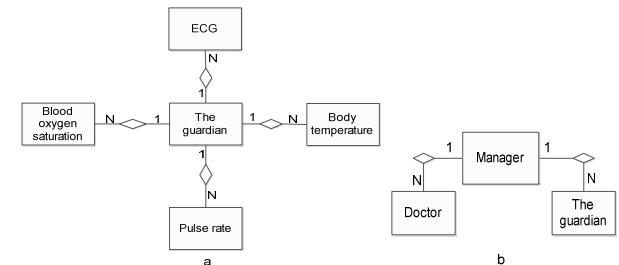

Figure 6. (a)The entity relationship of the guardian - physiologic information. (b) The entity relationship of the manager, doctor, and the guardian

\section{HEALTHCARE SYSTEM PLATFORM APPLICATION}

The guardian's operation will be demonstrated step by step at the beginning of logging in platform. Figure 7 is shown to give the login process about the guardian or doctor. Step A2, he first enter the information list interface in which particular types of files belonging to him are shown, for example ECG (as shown in Figure 8). Then he enter display interface, in which he can set the query period (as shown in Figure 9). He also could first enter the printing conditions controlling interface (as shown in Figure 10). When he input the appropriate information, he will get PDF document (as shown in Figure 11). Other query interfaces is not shown, only be listed about ECG for that the other interfaces are very similar to each other; step A4, if the guardian has any question about his monitoring results, he could communicate with the doctor online (see Figure 12).

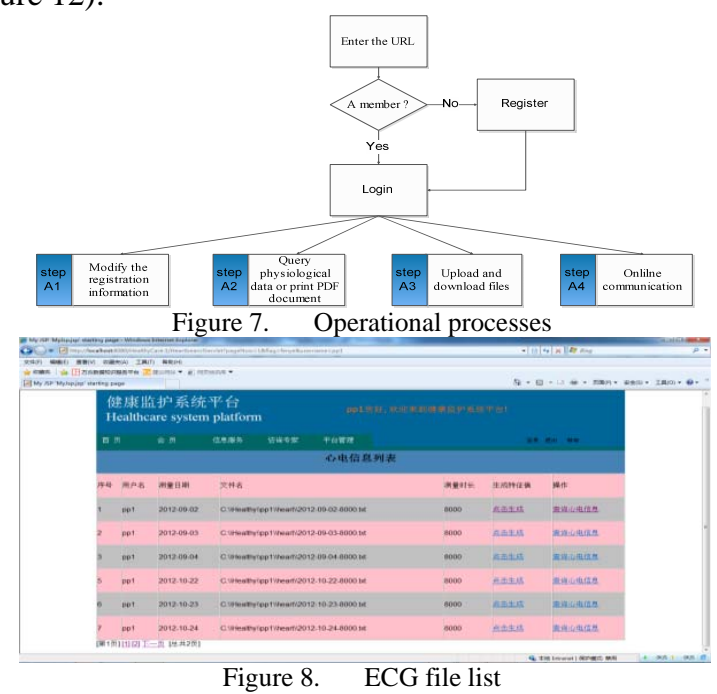




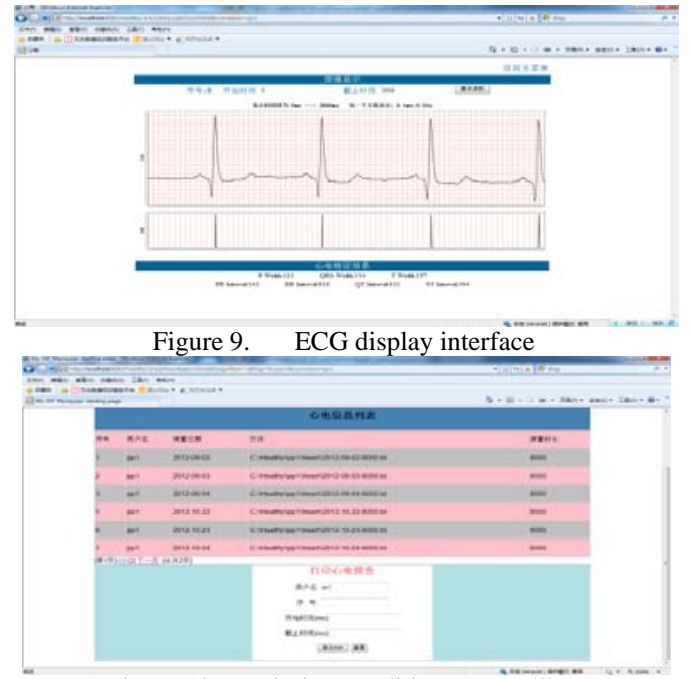

Figure 10. Printing conditions controlling

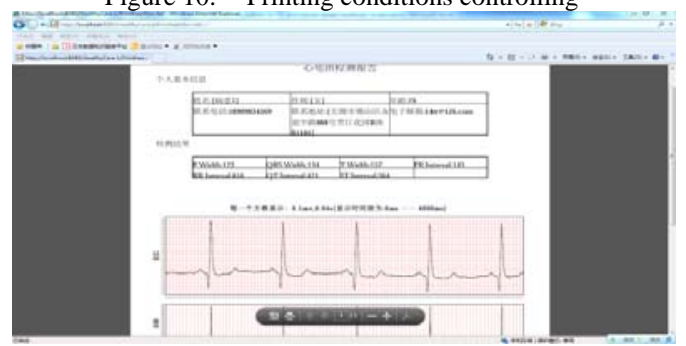

Figure 11. PDF document(ECG)

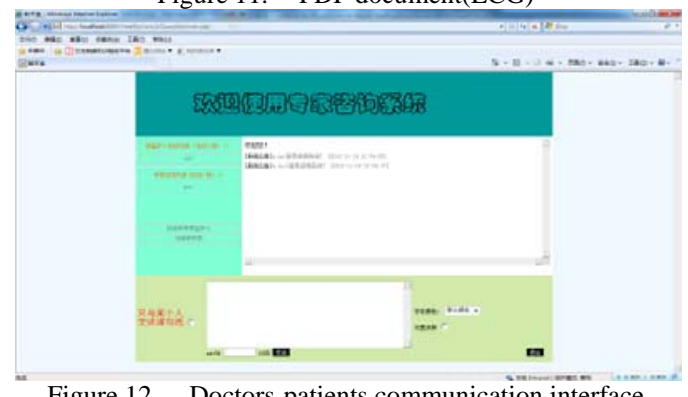

Figure 12. Doctors-patients communication interface

Doctor's operation is similar to the guardian's operation, so not to itemize here. But there is a difference that when the doctor queries physiologic data or print PDF document, he should input the username and select physiologic data types to search the user.

In Figure 13, the guardian physiologic data file was listed. The manager also can search the guardian that he wants to view the registration information.

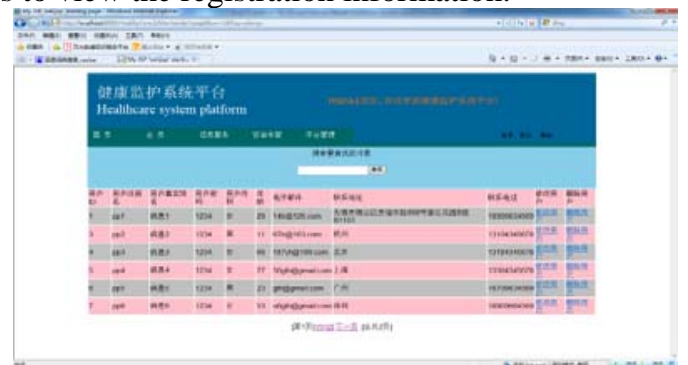

Figure 13. Management the guardian registration information

\section{CONCLUSION AND FUTURE WORK}

We have designed and implement the platform for 3G and Internet-Based Healthcare System. The guardian (patient or consumer) wear wearable physiologic monitors to get physiologic data such as ECG, blood oxygen saturation (SPO2) and body temperature. These detected data can be further upload to the server-side database through a smart phone. And then he could log in the platform, inquiry the physiologic data, print PDF report and consult doctor. The characteristics of this platform are: (1) The platform is Web-based and it is convenient for users to utilize this platform no need to install the client software, (2) The platform was build with free and open source Java library. The database server and web application server selected are also open source software. (3) The platform has not only physiologic data display function, but also has data analysis capabilities.

There are also some shortcomings inevitably. We use the wavelet transformation to process ECG data, but processing speed is slow. We will improve ECG analysis algorithm using second generation wavelet transform ${ }^{[9]}$ to change the design approach. We also hope that this platform has a diagnostic function automatically, and then it can reduce the work of doctors. Perhaps a lot of patients are more willing to communicate with doctor face-to-face and are not satisfied with only text exchanging. So it is necessary to implement video calling features.

\section{REFERENCE}

[1] Hu Zhan and Peng Xizhe, "China's Population Aging from the Perspective of Public Policy, " Social Sciences in ChinaVol. XXXII, No. 4, November 2011, 106-124.

[2] Liu Huawen, "Thoughts on Development and Ageing," HUMAN RIGHTS NO. 3 MAY 2009.

[3] Yundong Xuan, Zhan Zhao, Zhen Fang, Daoqu Geng and Yaohong Shi, "3ACare: A Wireless Body Sensor Network for Health Alert and Monitoring,” Advanced Materials Research Vols. 217-218 (2011) pp.1075-1080.

[4] Apache Tomcat, http://tomcat.apache.org/.

[5] Neal Ford, “ARTOF JAVA WEB DEVELOPMENT, Manning Publications Co.,” November 2003.

[6] http://www.jfree.org/jfreechart/.

[7] BRUNO LOWAGIE, “iText in Action Second Edition, Manning Publications Co.,” 2011.

[8] http://www.jdom.org/.

[9] Majdi Bsoul and Lakshman Tamil, "Using Second Generation Wavelets for ECG Characteristics Points Detection," IEEE Biomedical Engineering (MECBME), 2011 1st Middle East Conference on, 2011, pp. 375 - 378. 